\title{
From organic chemistry to fat and oil chemistry*
}

\author{
Etienne DEFFENSE \\ Crystallisation \& Degumming SPRL, \\ Terre l'Oreye 21, \\ B-6032 Charleroi, Belgium \\ $<$ e.deffense@crystallisation.be>
}

\begin{abstract}
With his work on animal fat and identification of fatty acids, Chevreul was a pioneer in organic chemistry. As Chevreul, I had a passion for organic chemistry too. It was then, an honour and a pleasure to present in 2008 at EFL in Athens this presentation entitled "From organic chemistry to fat and oil chemistry" because my background in organic chemistry helped me all along my professional career to understand and implement new developments related to oil and fat technology and processing. Among the topics which I worked out, I highlighted more particularly the following subjects: the degumming chemistry of oil and fat; the improvement of physical refining; a new chemical analytical tool for the dry fractionation; new development in the dry fractionation
\end{abstract}

Key words: oils and fats, degumming chemistry, physical refining, dry fractionation, crystallization, HPLC
It is not without any reason if I entitled the Chevreul's presentation: "From organic chemistry to fat \& oils chemistry". Michel Eugène Chevreul, born in 1786, was a pioneer in organic chemistry with his work entitled: "Chemical research on animal fats" (1823) and "General consideration on Organic analysis" (1824). Although the mineral chemistry was in vogue at that time, he was particularly well known for his work on fatty acids, showing that animal fat for example is composed of glycerol esters. He discovered fatty acids, among others: butanoic (C4:0), hexanoic (C6:0) and heptadecanoic acids (C17:0) which he named respectively butyric as derived from Butirum in Latin, capric because the smell reminded him the typical smell of goat dixit in Latin capra and margaric from the Greek Margarum meaning pearl in English, because the acid crystallised as a pearl. With Gay-Lussac, also known in Chemistry, he patented the preparation of candles from stearic acid, which was a new way to light without smoke.

Director of tincture at "Manufacture des Gobelins in Paris", Chevreul opened his own laboratory and published: De la loi du contraste simultané des couleurs where he showed the influence of colour to each other as an example, yellow near green becomes violet. At that regard, he influenced the French Impressionism.

As Chevreul, I had a passion for organic chemistry, especially for organic synthesis where the target has been to create new "synthons" in

\footnotetext{
* AFECG Chevreul Medal Lecture, said inside the 6 th Euro Fed Lipid Congress, Athens Greece, 8 september 2008
}

order to synthesise new molecules. As an example, in the Seventies, there was a tolerance and for some people an allergy to penicillin, therefore new organic compounds used as antibiotic have been synthesised such as cephalosporins and modified penicillins.

Later on, I had the opportunity to work in a new field, the organo-metallic chemistry, in a MaxPlanck Institüt in Germany to synthesise nickel or cobalt organic compounds used as catalyst in the polymers industry. When I joined S.A. Fractionnement Tirtiaux, in 1980, I came with my background in organic chemistry which helped me to understand better the fat and oils chemistry as shown all along my presentation. Early Eighties, the oil and fat field work was quite much empirical and most scientific research belonging to the two biggest companies Procter \& Gamble in USA and Unilever in Europe. At that time, all was kept very secretive and there was protectionism so it would be unimaginable to launch for example a new margarine labelled low trans fatty acids.

World has changed, consumers have become more aware of the quality of the food that they consume and most scientific information is available as well as on the net. For those interested in scientific papers related to this presentation, I shall invite them to consult all references via internet as I would have to mention here more than six hundred references.

In September 2008 at $6^{\text {th }}$ Euro Fed Lipid (EFL) Congress in Athens, I highlighted the scientific approach which has been taken and the impact this had on the case of implementation.

This paper will review briefly some selected topics which I worked out more particularly:

- the degumming of oils and fats and its chemistry;
- the improvement of the physical refining; - a new chemical analytical tool for the dry fractionation;

- new developments in the dry fractionation.

\section{Degumming chemistry and processing}

One of the key steps in refining process is the elimination of impurities from crude vegetable and animal fats, especially the phospholipids generally named gums. They are mainly present in vegetable oils extracted with solvent. These compounds are similar to, triacylglycerols (TAGs) or triglyceride molecules but differ in that one carboxylic acid or fatty acid chain is replaced by a phosphate group that may be linked to different substituents. Among main phospholipids, we may list phosphatidyl choline (PC), phosphatidyl inositol (PI), phosphatidyl ethanolamine (PE), phosphatidic acid (PA) and calcium, magnesium, iron salt of $\mathrm{PA}$ i.e. CaPA, MgPA and FePA. Due to the presence of two long lipophilic fatty acid chains and a hydrophilic phosphate group on the same molecule, the phospholipids behave like emulsifiers. Among them PC and PI being more hydrophilic are considered as hydratable phospholipids compared to PE, PA, CaPA, MgPA, FePA which are named Non Hydratable Phospholipids (NHP).

Due to their emulsifier properties, they are responsible for oil loss in refining process and due to the fact that they decompose under heating, darkening the oil, phospholipids have to be eliminated in the early refining stage. For fats obtained by pressing i.e. poor in phospholipids, like animal fats, palm oil, palm kernel oil, 
olive oil..., the degumming process looks quite simple. It consists to mix the fat with a small quantity of degumming acid such as phosphoric or citric acid to dissociate the NHP left mainly in the fat. Then the new species as well as calcium or magnesium bi-phosphate are immediately adsorbed on bleaching earth and eliminated by filtration (figure 1).

When trying to understand the organic chemistry of such chemical reaction, apparently simple, applied in industrial scale for palm oil in Malaysia as "dry degumming" process, in the Eighties, I discovered two things. First, most plants used calcium carbonate afterwards to neutralise the excess of phosphoric acid probably to protect the equipment against the corrosion. I thought that calcium should reverse the initial reaction leading to calcium salt of phosphatidic acid (CaPA). Analysis of dry degummed bleached palm oil samples collected from twenty refineries confirmed that hypothesis: the average content in phospholipid measured as phosphorus was 15 ppm against 20 ppm for crude palm oil.

Furthermore, some refiners believed that excess of phosphoric acid would eliminate phospholipids even better, without noting that excess of phosphoric acid left in oil after bleaching would catalyse the hydrolysis of triglycerides at high temperature during physical refining, increasing the loss in oil but also forming new phospholipids species by reaction of phosphoric acid with diglycerides DAGs. Nowadays, having a better understanding of the chemistry, most refiners are either using degumming acid like phosphoric acid with moderation or neutralise excess with sodium hydroxide but still there are plants using calcium carbonate in 2000.

When going further into degumming chemistry of oils rich in phospholipids, especially in NHP, it is clear that "dry degumming" could not be applied unless using a huge quantity of earth. Conventionally, for such oils, the best way to make the NHP hydratable is to raise the $\mathrm{PH}$ by addition of an alkali in order to shift the equilibrium towards a hydratable sodium salt. This is what is taking place in the chemical caustic refining process where all fatty acids are eliminated with too.

Early Eighties, most degumming processes, among them the super degumming, patented by Unilever, relayed on multi mixing with citric acid, incorporating cooling stage but results were quite dependent on oil quality.

Only end of the Eighties came the "special degumming" processes based on partial neutralisation of phosphatidic acid by sodium hydroxyde without touching the free fatty acids (figure 1).

Furthermore, we have investigated the purification of crude palm oil by an enzymatic

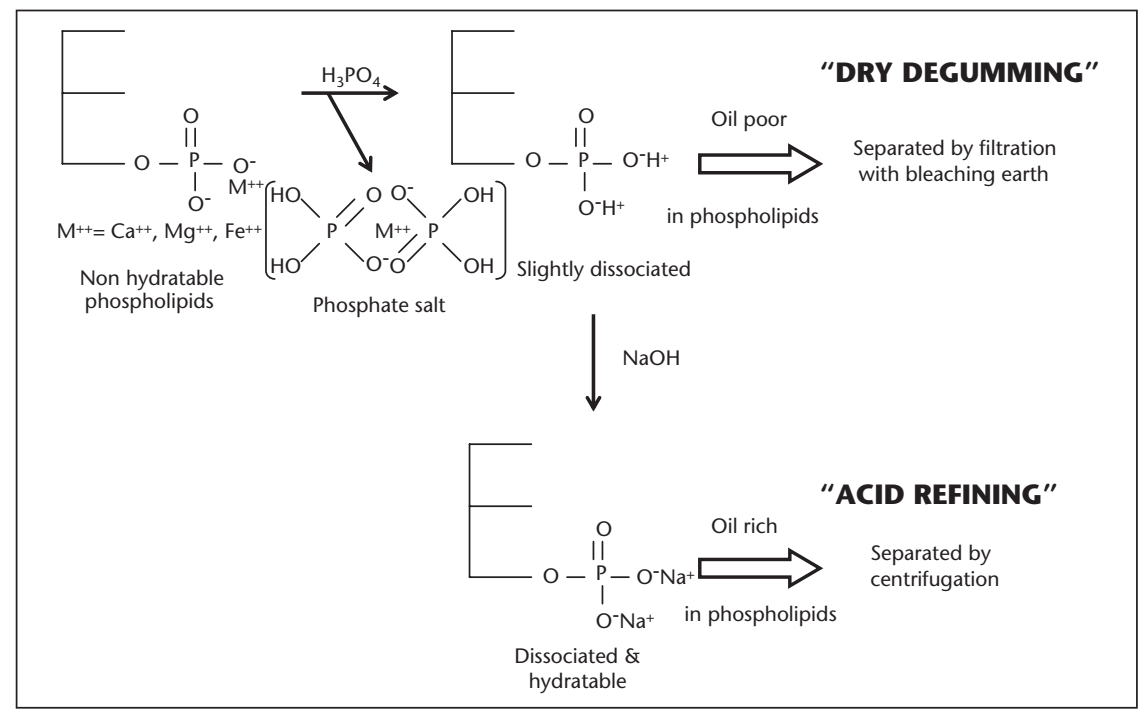

Figure 1. Chemical degumming routes

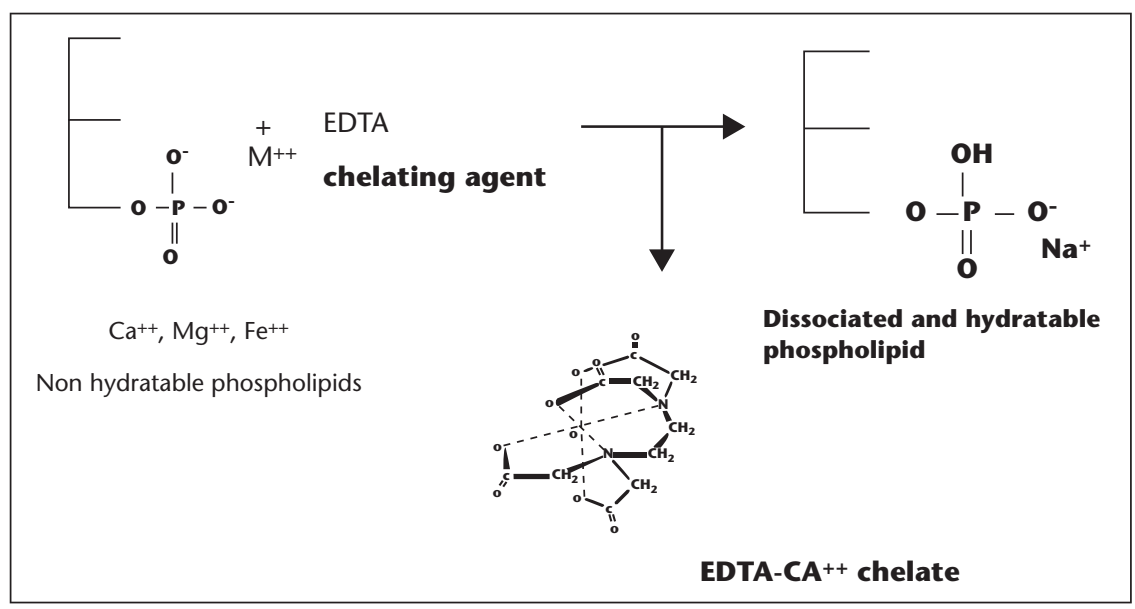

Figure 2. EDTA Degumming. "Soft degumming" (1995) PCT E. Deffense et al. "Complete degumming" (1998) PCT E. Deffense

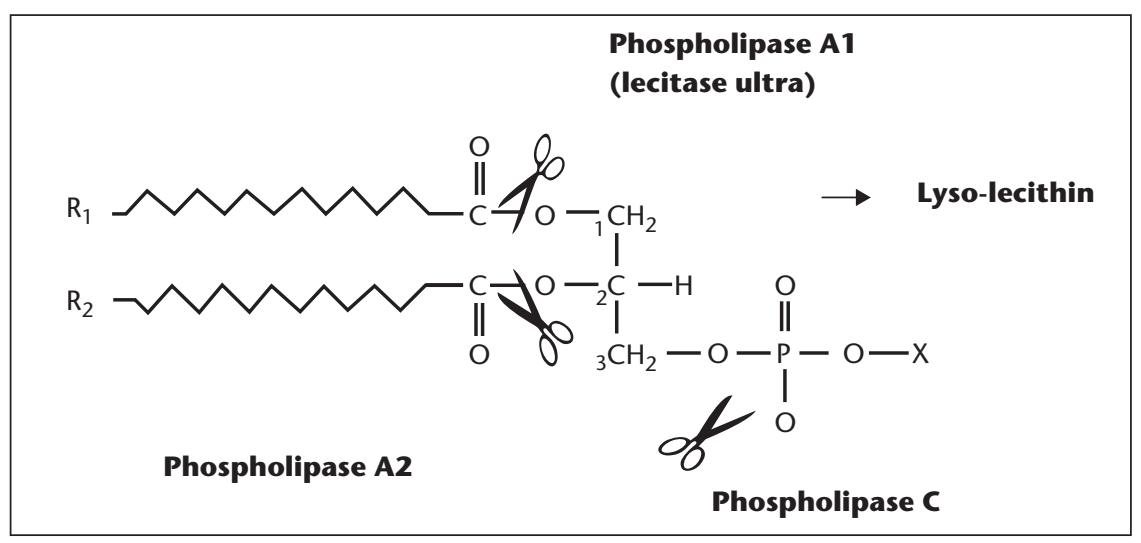

Figure 3. Enzymatic degumming 


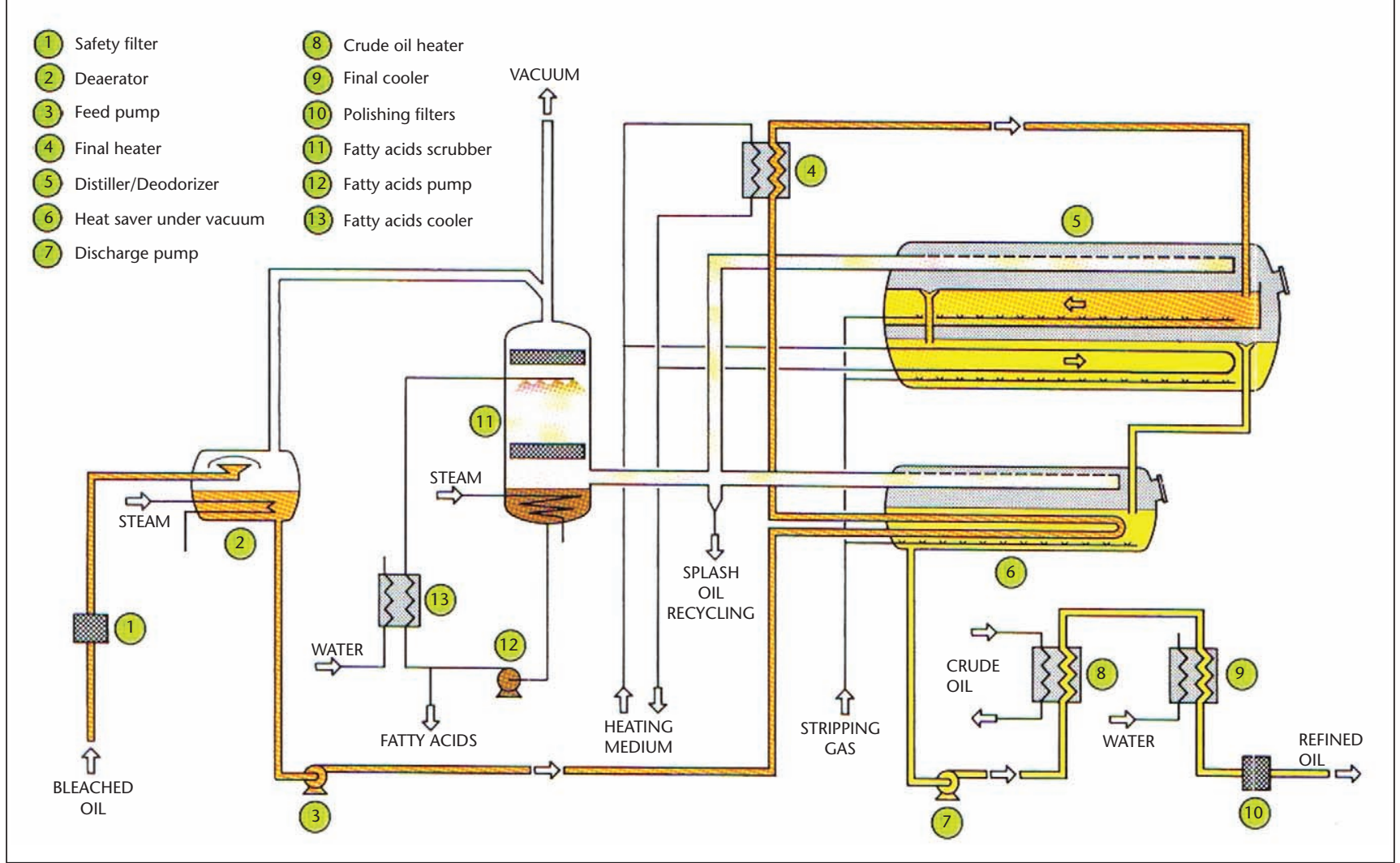

Figure 4. Shallow bed deodorising plant: Lau's system.

route to improve the crystallisation. The process consisted to treat palm oil with pectinase and cellulase in order to eliminate pectin, cellulose and fibbers (EP0 070269 A2 Patent 1982). The process has been applied with success. Later on another company patented an enzyme assisted aqueous extraction of olive oil to obtain better yield. Nowadays, the former process is applied accordingly for other oils. Based on our first experience on enzymatic treatment and although nothing was published on oil enzymatic processes, early Eighties, we investigated the "dephospolipidation" of NHP of soybean oil using phospholipase C. Available only in small scale, the production of such enzyme in big scale looked like a challenge for most suppliers. Furthermore to use enzyme for bulk oil processing seemed also unrealistic. Thanks to Amano in Japan, the enzyme has been made available. The purpose was to split the phospholipid into a DAG and a hydratable phosphate molecule as shown in figure 3. Using a water degumming soyabean oil containing 200 ppm as phosphorus, it has been shown that most phospholipids were eliminated easily, except phosphatidic acid salts which have never been removed completely: 15 to $20 \mathrm{ppm}$ as phosphorus were always registered.
As for most enzymatic treatments, additives were used to facilitate the hydratation and then the contact between the enzyme and the phospholipid. Different chemicals have been tested among them, sodium lauryl sulphate (SLS) as detergent and a complexing

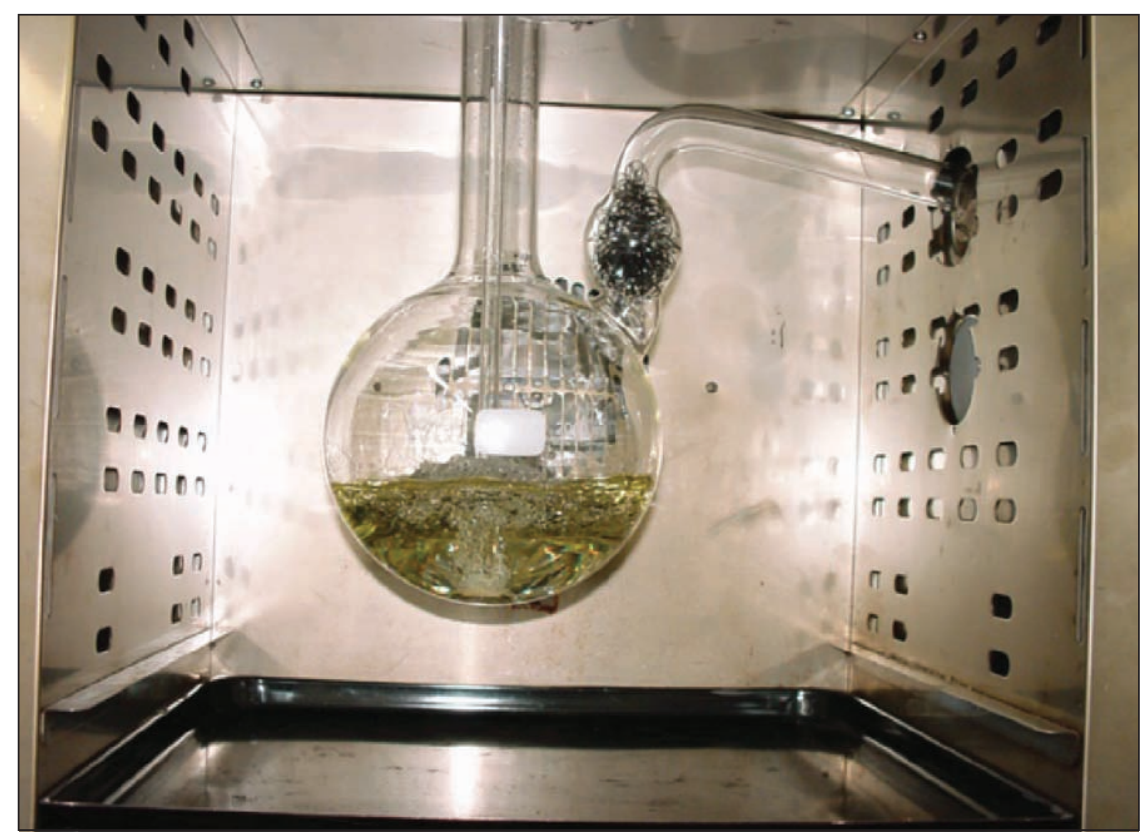

Figure 5. Turbo deodoriser pilot plant 
agent to capture the metal ion, $\mathrm{Ca}, \mathrm{Mg}$ and Iron. Among these organic acids, citric acid gave a good result but ethylene diamine tetracetic acid (EDTA) used as chelating acid, gave an excellent result: phosphorus level was always lower than 2 ppm after reaction.

Going further in the research, it was observed that elimination of phospholipids took place even without the phospholipase $C$. This was explained by the fact that the divalent metal ion was chelated immediately by EDTA forming a much stronger complex with the ion. Consequently the new species, the phosphatidic sodium salt became hydratable. The detergent SLS makes easier the contact between the phospholipid molecule in the oil phase and the water phase solution containing the chelating agent. This process has been patented under the name of "Soft Degumming" PCT patent 95/006009 (1995) E. Deffense et al., but one of the drawbacks of that process was the high stability of the emulsion making the separation difficult (figure 2).

In 1998 Crystallisation \& Degumming (C \& D) patented the "Complete degumming" using EDTA without SLS; the results were as good as those obtained by the "Soft Degumming" but the separation of the oil phase from the water phase was much easier because the new process consisted to generate a temporary emulsion phospholipids-EDTA using the hydratable phospholipids present in the oil as emulsifier. As a result, lower oil loss by entrainment was observed during the separation. The overall refining yield obtained with soyabean oil or rapeseed oil was on average $1 \%$ higher than that obtained with caustic refining process. The process has been implemented on industrial scale up to 500 tons per day for soyabean oil and rapeseed oil.

\section{What is the future of the degumming processes?}

On one side, a number of special degumming processes grouped on the name "Acid refining" (figure 1) have been developed in the Nineties, driven by the industry to enable the physical refining of seed oils. Compared to chemical refining, there is no soapstock and much less oil loss by entrainment. Latest degumming process launched in the market was the enzymatic degumming using either phospholipase A2 or A1 generating a hydratable lyso-lecithin plus one free fatty acid molecule. It was a mild treatment but still requesting chemicals to buffer the reaction, a long reaction time - minimum four hours - and an enzyme which was not cheap. Compared to caustic refining, there was $1 \%$ overall yield higher like for the "complete degumming".
In 2008, Dayton published and patented a new enzymatic degumming process. Previously, he also observed that phospholipids cannot be completely removed or split by the phospholipase $C$ as we observed twenty years earlier but he found and highlighted a synergistic effect

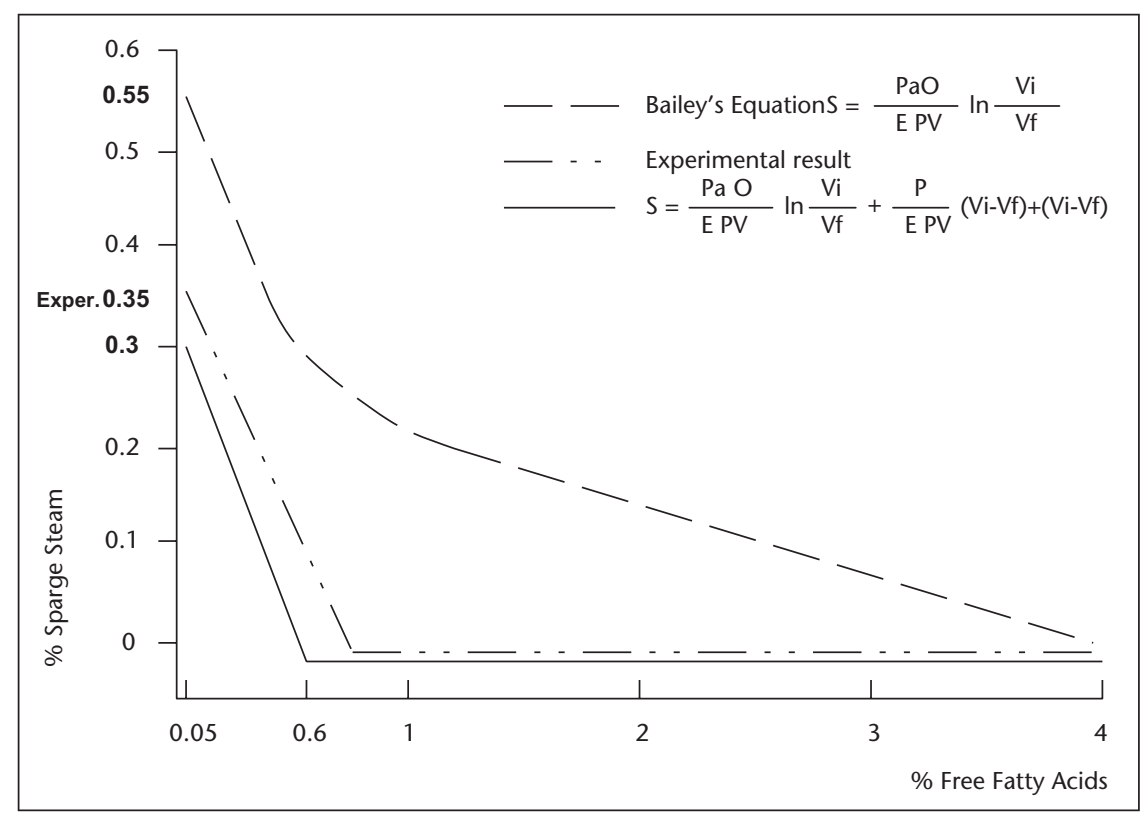

Figure 6. Stripping steam consumption versus fatty acid reduction

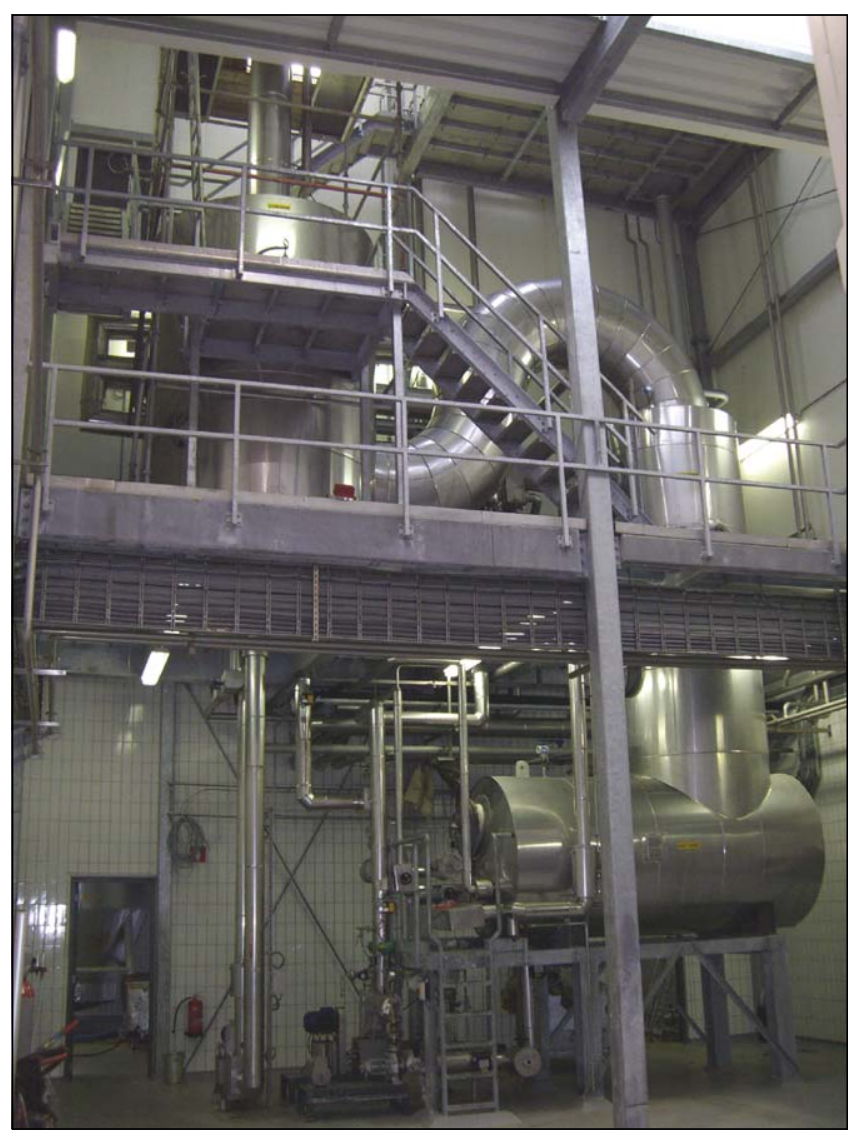

Figure 7. $C \& D$, Packed column deacidification plant 
between phospholipase $C$ and phospholipase A1: using both enzymes together it reduced drastically the reaction time to only $30 \mathrm{~min}$. And as the reaction was fully complete, almost $2 \%$ overall yield was obtained.

Trends in degumming will depend on many options, namely on the lecithin recovery or not, on cost effectiveness, environment aspect (pollution), yield (loss of oils), gums disposal for example via the meal or not, cost of enzyme and of the licence as well.

Organic chemistry remains always present in the chemistry of oils and fats. Beside the degumming chemistry, other subjects have been investigated. For example, formerly with the development of physical refining via the packed column, degummed bleached palm oil used to be overheated to overcome the heat loss from the evaporation of fatty acids. Hence $\alpha, \beta$ unsaturated aldehyde or acrolein, has been generated by decomposition of glycerol at high temperature namely by dehydratation of glycerol in acidic conditions.

More recently, the presence of other organic chemicals found in refined vegetable oil requires our understanding. For example, the presence of 3-monochloropropane-1,2-diol fatty acid esters (3-MCPD esters) and 3-monochloropropane-1,2-diol (3-MCPD). They appeared to be formed at high temperature in presence of chloride ion but many questions remain open on the reaction pathways and conditions leading to the formation of 3-MCPD esters.

\section{The physical refining improvement}

Having in hands degumming processes suitable for oils with high content in phospholipids, and our aim has been to understand and improve the physical refining process.

Described for the first time in 1866, as a kind of purification of palm oil with superheated steam, to extract colourless fatty acids from reactor made of copper, it was indeed the principle of steam distillation used nowadays for physical refining.

For reminder, the aim of physical refining is multi-purposes:

- to eliminate all undesirable odoriferous volatile substances such as flavours, oxidation products, pesticides and free fatty acids which have a negative effect on taste, appearance and shelf life;

- to decompose, if still present, peroxides, temperature sensitive colouring matters andpigments such as carotene;

- to reduce oil loss by entrainment by steam stripping as well as elimination of natural antioxidants such as tocopherol;

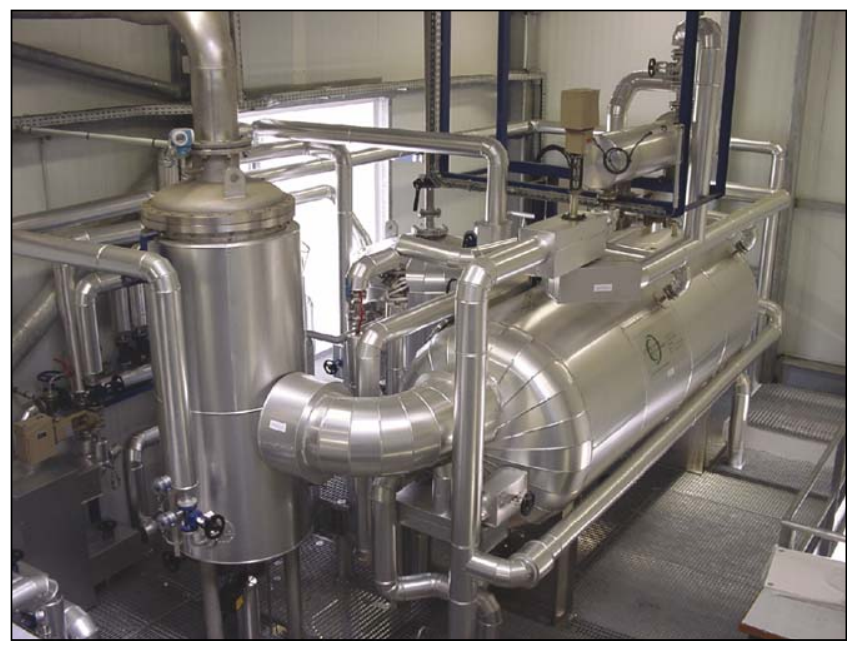

Figure 8. C\&D, shallow bed horizontal batch deodoriser $1000 \mathrm{~kg}$ (ITERG)

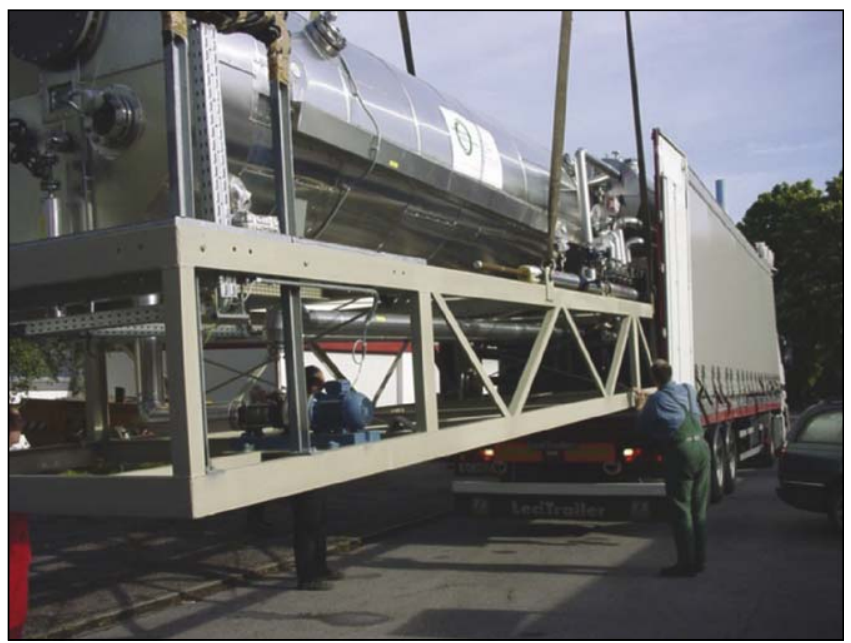

Figure 9. C \& D skid mounted horizontal deodoriser plant for fish oil

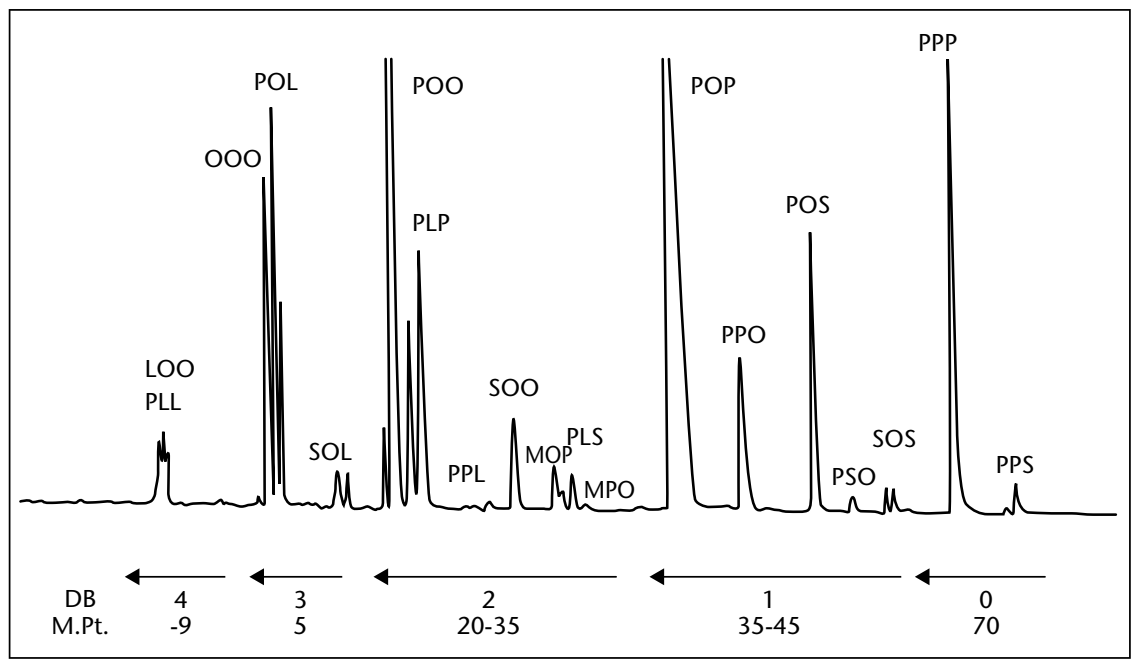

Figure 10. Positional 1-2, 1-3 isomers separation of palm oil by RP-HPLC 
- to avoid formation of new compounds such as polymers and trans fatty acids (TFA);

- and last and not least at the lowest cost as possible in terms of energy, steam stripping and motive steam request.

Early Eighties, I have been asked to study the Lau's horizontal deodoriser (figure 4) with the inventor of the patent and examine the performances. The Lau's deodorizer comprises a series of horizontal cylindrical vessels with upper and lower deck with hot oil flowing from one side to the other in a shallow bed. Stripping steam is injected by multiple pipe distributors running all along the length of each deck. Vapours from different compartments are collected also all along the vessel. A bank of heating tubes compensates for the heat losses due to evaporation and radiation.

Looking at a cross section, we have a large exposed surface to vacuum; the sparging steam is finally distributed for better stripping efficiency and low energy consumption. Top layer is constantly renewed as the oil is moved by the sparging gas. The head space, being very large, the speed of distilled vapours is very low and minimises oil losses by entrainment.

A series of trials have been then conducted in a turbo deodoriser pilot plant, designed more or less as the one shown in figure 5 which is used presently in our pilot plant facilities at C \& D.

The research was aimed to answer the following questions:

- what is the minimum stripping steam requirement for deodorisation and physical refining; - what is the theoretical stripping steam consumption;

- which is the ideal layer of oil;

- how to heat the feed oil entering the physical refining plant with exchangers or under vacuum;

- how to cool down the refined oil after physical refining with regard to the oxidative stability of the oil: with or without stripping steam, under vacuum or not.

Among all these points which have been investigated in detail, let's review the comparison between the theoretical and practical stripping steam consumption obtained when conducting palm oil physical refining (FFA 4\%) at $260^{\circ}$ and 4 mbar in the turbo deodoriser.

The elimination of reflux and radiation losses combined with the optimisation of the stripping steam distributor reduced the necessary steam consumption significantly and resulted in a vaporisation efficiency factor $E$ of 0.93 compared to 0.6 for a standard refining plant. When comparing the experimental steam consumption value $0.35 \%$ (figure 6) with the theoretical value $0.55 \%$ using the Bailey's equation where the stripping steam quantity is proportional to the operating pressure in the deodoriser in mbar [Pa], quantity of oil in moles [O], inversely proportional to the vapour pressure of pure fatty acids at the operating temperature $260{ }^{\circ} \mathrm{C}$ ( $P V=72 \mathrm{mbar}$ ) and also inversely proportional to the vaporisation efficiency (factor $E=0.93$ ), there is a discrepancy. The experimental duplicated results were always much lower than that obtained by Bailey's equation where $S$ is the total moles of steam injected, Vi the moles of initial fatty acids and $V f$ the moles of final fatty acids. This has led to revisit the calculation of the theoretical quantity of steam applying the Dalton's and Raoult's laws which resulted in a totally different equation with a steam value of only $0.30 \%$ almost half value of that obtained from
Bailey. It means that the Bailey's equation which has been mentioned and used as such for 50 years was only valid for deodorisation when initial FFA content is so low that the full equation can be simplified hence, for physical refining the two right hand terms must be included.

When looking at the curve drawn from the new theoretical equation or full equation, there is a straight lime between 4 and $0.6 \%$ FFA meaning that there is a spontaneous distillation of free fatty acids down to $0.6 \%$. Experimentally it has been shown that no steam was required to deacidify palm oil from $4.0 \%$ down to $0.8 \%$.

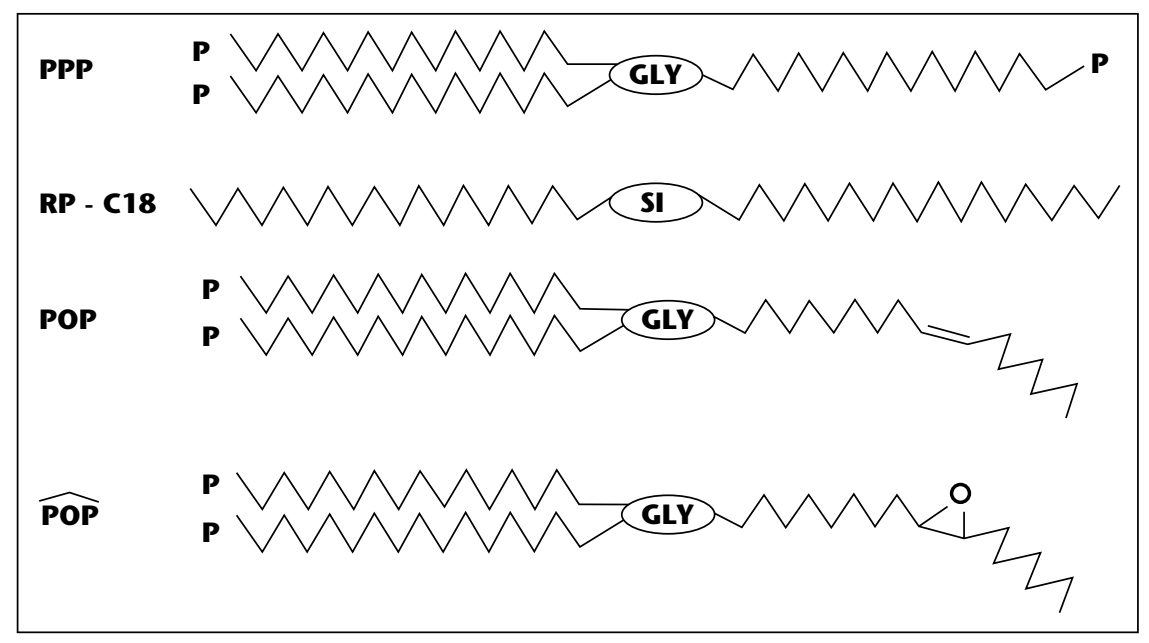

Figure 11. RP-C18 affinity with PPP,POP and PÔP

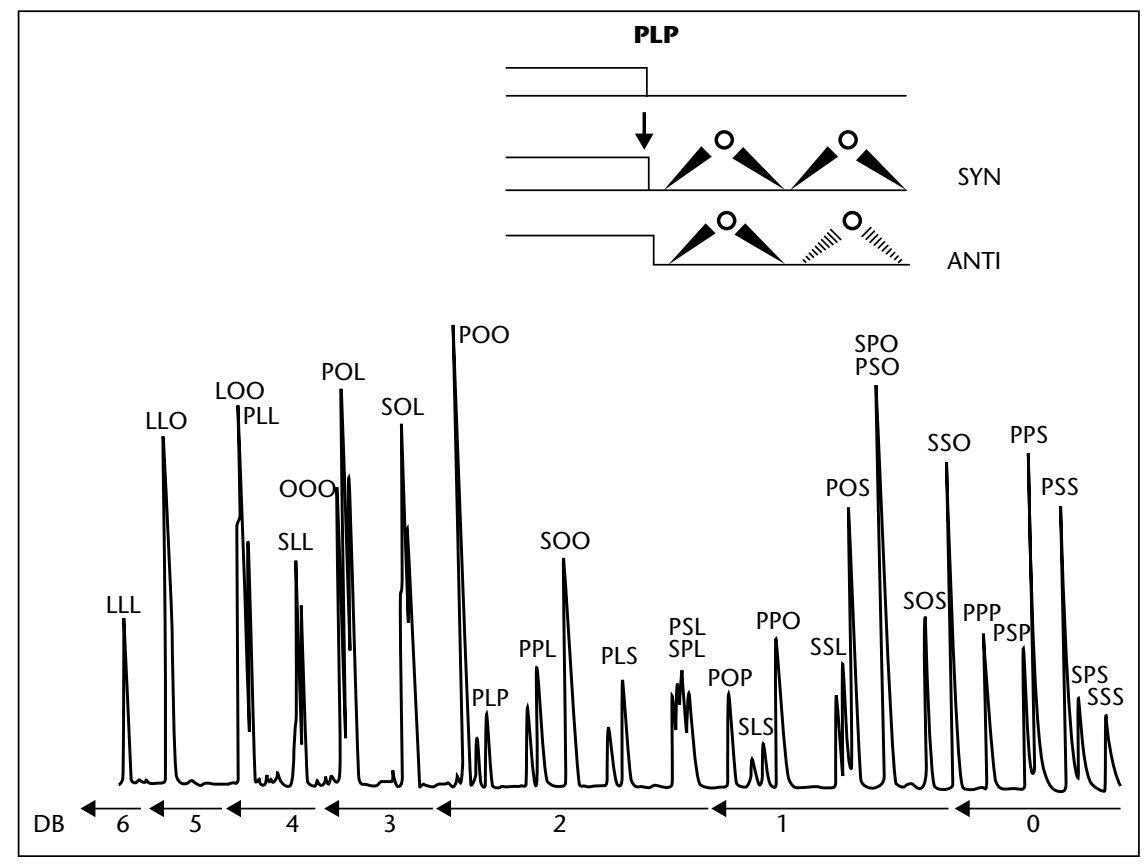

Figure 12. RP-HPLC chromatogram at $5{ }^{\circ} \mathrm{C}$ of a randomised, epoxidised mixture TAGs PPP-SSS-000-LLL. 
The fact that it was proved that we do not need any stripping steam during the first step of physical refining and that with fine stripping steam distributor combined with shallow bed, the best performance could be obtained, it was possible for example to design multihorizontal vessels plant of 400 TPD capacity to reduce FFA from $5 \%$ to $0.04 \%$ with only $0.6 \%$ sparge steam. As a consequence, less stripping steam consumption meant less motive steam consumption for the vacuum unit, less hydrolysis of TAGs and less oil loss due to entrainment.

The stripping steam consumption is comparable to value obtained with packed column (figures 7 C \& D 200 TPD packed column plant (2006)) because if we do not need much steam to deacidify for the reason explained above, we still need some stripping steam to complete and deodorise the oil where some new molecules resulting from the cracking at high temperature need time to be eliminated with sparge steam.

Latest developments made by $C \& D$ these last 10 years comprise the application of the horizontal tray deodoriser (HTD) for special applications where normally a high quantity of steam is needed: for example, the stripping of cholesterol from milk-fat for which a minimum stripping steam was necessary. Low cholesterol butter produced by steam stripping is till on the market in Germany. Parallel to that crude pretreated palm oil has also been deodorised to produce Golden palm oil rich in carotene, tocopherols and tocotrienols. Plants are running in Malaysia and South America.

With regard to the high efficiency and lower stripping steam requirement, the technology has also been adapted for stripping the PCB and dioxins from fish oil to meet EU and USA requirements namely to reach less than 2 PPT (C\&D, 2005, 150 TPD plant).

In the same trends, the horizontal batch deodoriser being also much more efficient than the vertical batch, with less than 2 hours against 4 to 5 hours hold-up time, it became suitable for any kind of fat, oleochemical and especially for very sensitive products. Small shallow bed horizontal batch deodorisers from 300 to $2000 \mathrm{~kg}$ have been designed (figure 8) with following advantages: low capital cost, simple process, easy feedstock change over, low contamination, low stripping steam consumption and short hold up time.

Parallel to that the horizontal deodoriser has been designed as compact skid mounted shallow bed deodoriser from 5 to 100 TPD (figure 9). Combining at the same time, the batch and continuous on the same skid with energy recovery, the aim is to proceed physical refining or deodorisation of vegetable oils or soft refining of bio-vegetable oils or bio-organic oils as well.

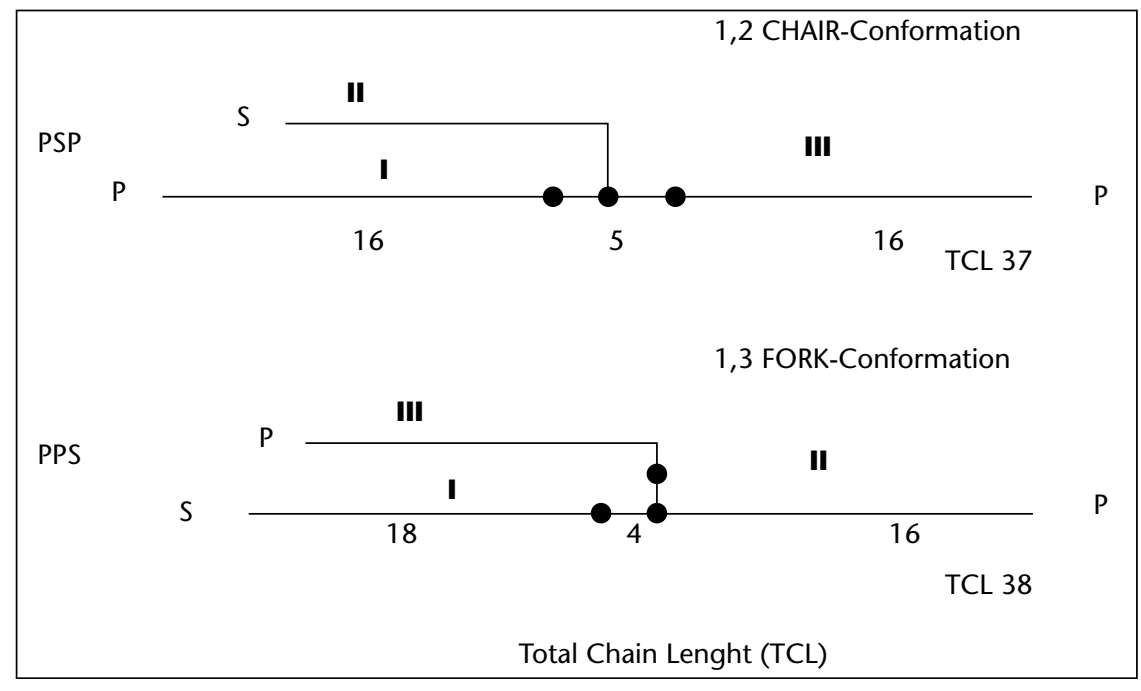

Figure 13. PSP-PPS Conformations

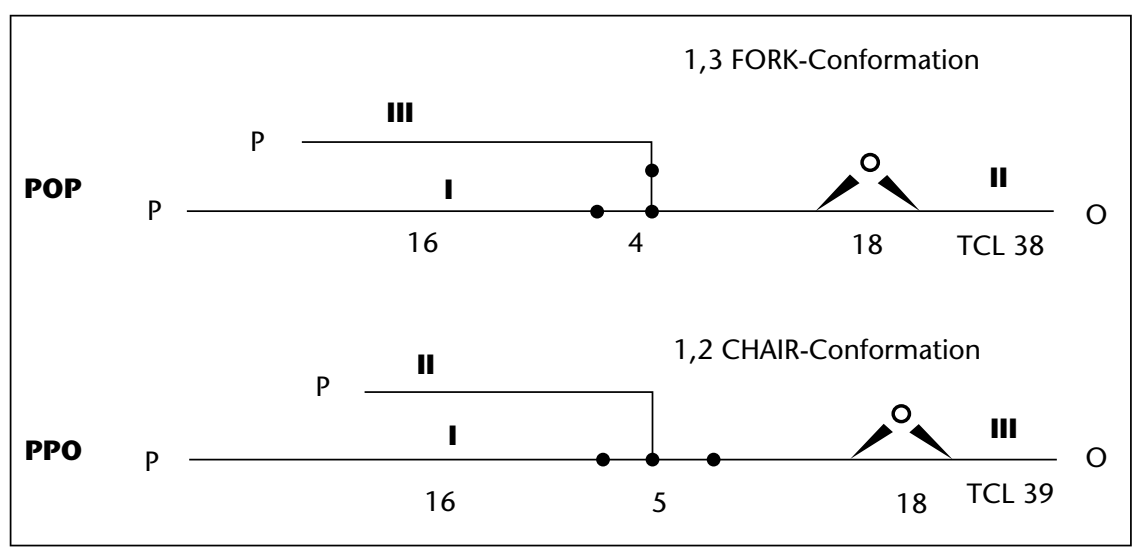

Figure 14. Conformation of epoxidised PÔP-PPÔ.

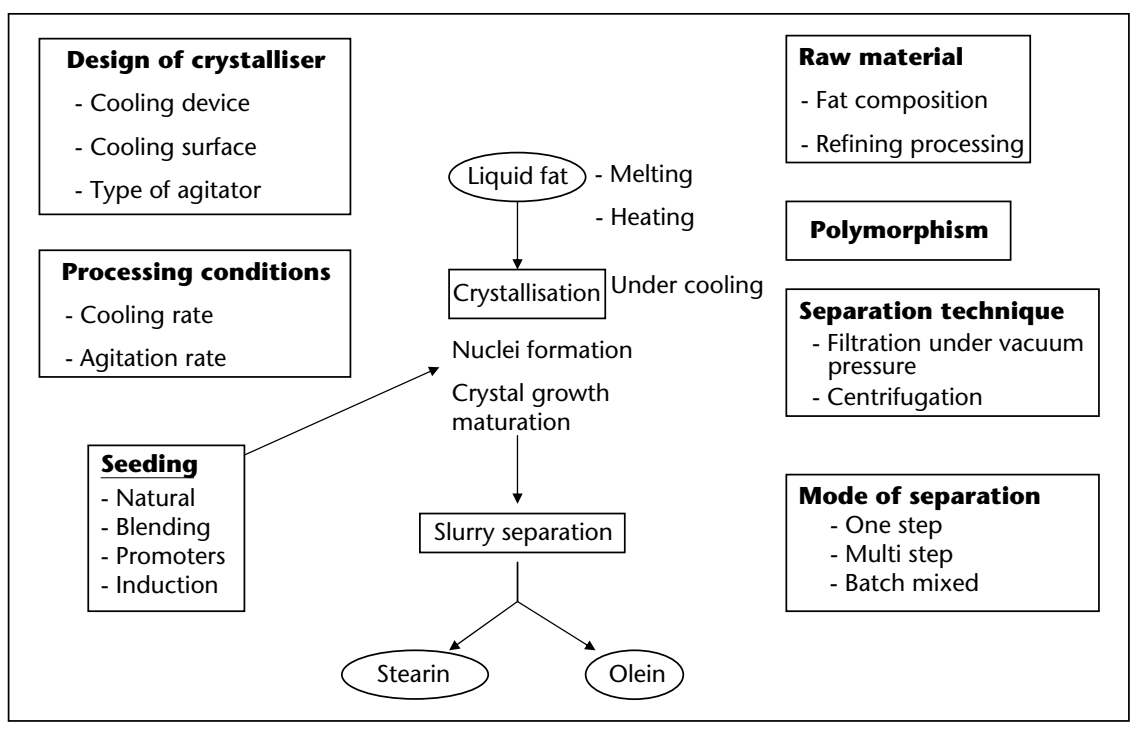

Figure 15. Dry fractionation schema 
The "skid mounted" horizontal deodoriser offers many advantages: less time from date of order until start up, less cost of freight, less cost for total erection, insulation, tests, supervision of erection done by experienced engineer as well as pre-tests, simple building needed, just to connect product in and out, water, steam, electricity, condensate and return water out, just to be placed on the ground floor.

\section{Dry fractionation and analytical tool}

Going further in oils and fats modification by dry fractionation, it was particularly crucial to hold new analytical tools for monitoring the dry fractionation process. A better knowledge of the TAGs composition of fat should aim at a better understanding of the fat crystallisation. In the Eighties, palm oil TAGs analysis by reversed phase high performance liquid chromatography (RP HPLC) was a real challenge because the high melting trisaturated TAGs crystallise or solidify in the HPLC column. It has been possible to elute at up to $50^{\circ} \mathrm{C}$ and obtain an excellent analysis of palm oil and its stearin fractions. Since that time ovens for HPLC column have been available on the market and the column temperature has been always mentioned.

Although a good TAGs separation was obtained, none of positional isomers POP and PPO for example, was separated from each other. In Nineties, with the development of cocoa butter equivalent (CBE) made from Palm Mid Fractions (PMF), the identification and quantification of such isomers became crucial.

Here again, the organic chemistry background was necessary to imagine and find a way to differentiate the two isomers based on the position in 1 or 2 of the monounsaturated fatty acid chain on the glycerol. Three parameters have been investigated - the choice of eluent, the control of the column temperature and the derivatisation of double bonds of TAGs by epoxidation - to provide a separation of positional isomers POP-PPO, POS-PSO as shown in figure 10.

Looking at figure 10, it is clear that tripalmitine (PPP) has the largest retention time due to the excellent affinity of palmitic acid chains with the octadecyl bonded phase of the column (figure 11).

Compared to PPP, POP with one double bond increasing the polarity and modifying the linearity of the molecule, will have a lower affinity for the RP-C18. Accordingly, the cis-epoxystearic acid chain with the addition of an oxygen atom on the double bond increases greatly the polarity and creates also a stearic effect through the oxiran ring which decreases signi- ficantly its affinity for the stationary phase and thus explains the much shorter retention time. In the light of this observation, a mixture of TAGs PPP-OOO-SSS-LLL has been randomised and epoxidised to generate all the isomers met in CBE production.

Accordingly figure 12 shows that all TAGs are eluted according to their degree of unsaturation from zero (0) to six (6) double bond (DB). Within each group, TAGs are separated by chain length and according to their isomeric configuration, position 1-2 or 1-3.

The question is how we can explain the separation of PSP from PPS or PSS from SPS, all dissymmetric saturated TAGs of zero group without epoxidation. If we analyse the literature, we observe that PSP has preferentially the 1,2chair conformation with fatty acids on glycerol positions 1 and 2 adjacent, with the chain on the 3-position forming the back rest of the chair while PPS has the 1,3 tuning fork conformation. Looking at the two conformations (figure 13), the Total Chain Length (TCL) of PSP containing 37 atoms being shorter than that of PPS having 38 atoms, PSP elutes first from the RP column maintained at $5^{\circ} \mathrm{C}$. Accordingly, the same findings are valid for dissymmetric saturated TAGs PSS and SPS as well as for natural TAGs POP and PPO.

Compared to PSP, POP or PÔP has preferentially the 1, 3-tuning fork conformation and PPO or PPÔ the 1, 2-chair conformation (figure 14) but as POP has a TCL of 38 atoms compared to PPÔ with 39 atoms, PÔP will elute first in the same operating conditions.

It means that we can assume that all these TAGs are present in the column as liquid crystals when running the analyse at $5{ }^{\circ} \mathrm{C}$ with that eluent. In such analytical conditions all positional isomers 1-2 and 1-3 appear as twin peaks. However for monounsaturated TAGs, it was possible to split completely the twin peaks into two well separated peaks by epoxidation of double bonds (figures 10 and 12): the delta retention time between epoxidised diunsaturated isomers is much higher. Moreover all epo-

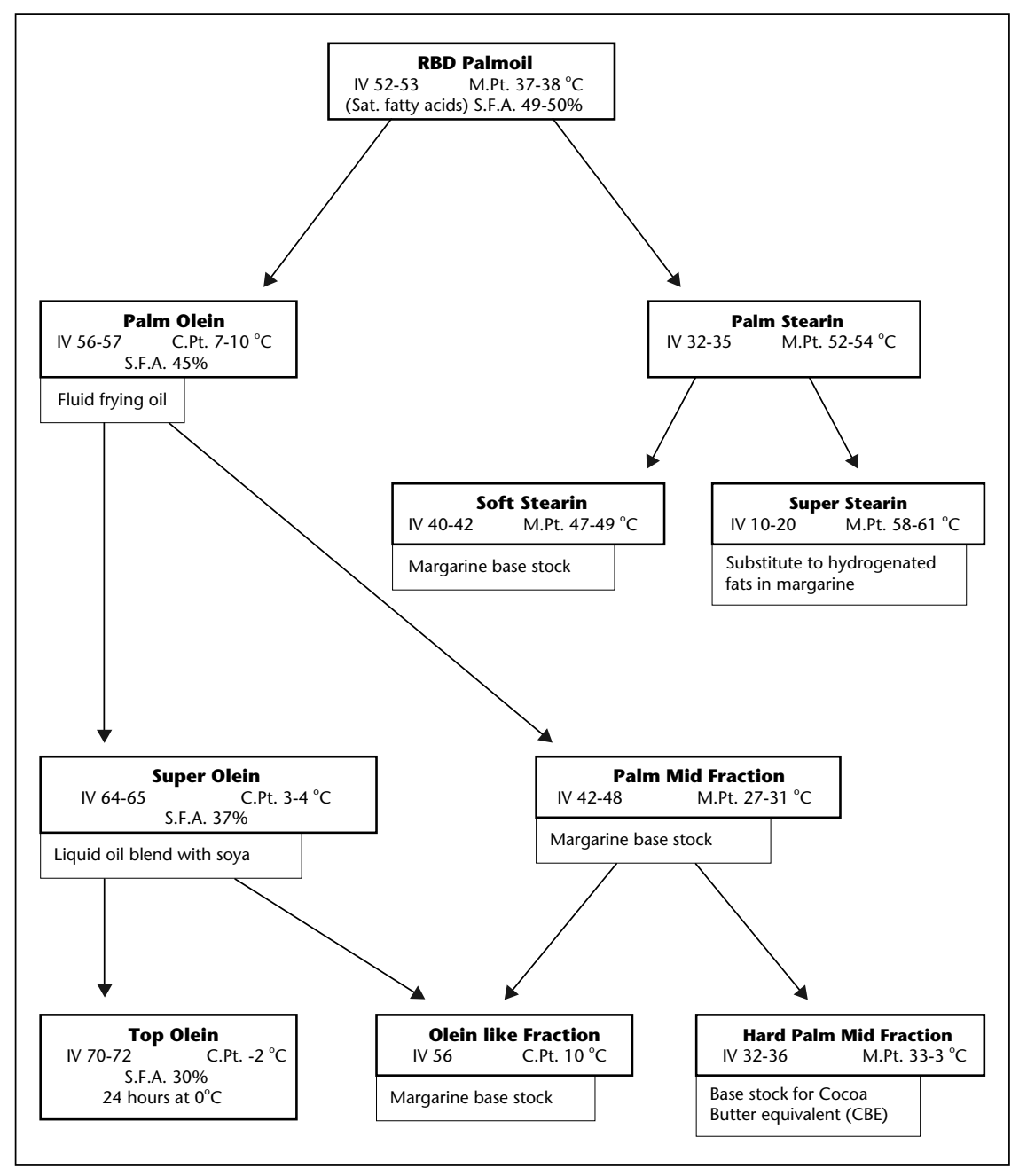

Figure 16. Multi steps fractions from palm oil dry fractionation 
xidised diunsaturated TAGs PLP and PPL show two additional separated peaks for each isomer because each isomer has two diastereoisomers Syn and Anti as shown on figure 12.

From the RP-HPLC analysis we observed that it is possible to extrapolate the conformation, type fork or chair of TAGs isomers. Same observation has been made with TAGs containing myristic acid as well as elaidic acid present in cocoa butter replacers (CBR)

This analytical tool enables to:

- monitor the PMF and HPMF production and control their quality for CBE application;

- determine the oil \& fat composition of:

- new specific fats and raw materials,

- genetically modified oils (GMO) too;

- check the purity of TAGs [JAOCS 75,285 (1998)];

- check the purity of fats and their adulteration:

- the molecular profiles of TAGs for detection of oil adulteration,

- the TAGs structure for the physiological properties;

- monitor the fat and oil processing:

- chemical and enzymatic interesterification,

- TFA formation during physical refining.

\section{Dry fractionation and new developments}

Early Eighties almost nothing was published on the crystallisation from the melt or on fractional crystallisation generally named dry fractionation of oils and fats. Theoretical aspects of the crystallisation and their implication for industrial fractionation have been investigated.

From a description point of view (figure 15), the fat is first melted completely and heated to eliminate any crystal memory. Then the melted fat is cooled under controlled agitation rate and cooling rate to generate the first nuclei from high melting TAGs. These nuclei grow to form crystals and then when the crystallisation has progressed far enough, under cooling which is the driving force, to allow the crystals to grow, mature and agglomerate, the crystals slurry is separated: the crystals or solid fraction named stearin is separated from the liquid fraction, the olein.

In practice the crystallisation process is not simple because it depends on many factors and parameters which are important to remind here (figure 15).

The type, the origin and the quality of the raw material hence the TAGs composition of the fat are factors affecting the crystallisation. The presence of minor components especially DAGs play a significant role in the crystallisation, for example, of palm oil and its fractions.

Conditions to obtain a good crystallisation relay on the polymorphism hence the composi- tion of the fat and the type of processed fat namely crude fat, pre-treated or fully refined fat. On that regard, in the Nineties we have highlighted the choice of bleaching earth and its effect on the dry fractionation and namely on the olein yield obtained.

The crystallisation is an exothermic reaction and oil being a poor conductor, the efficiency with which the heat of crystallisation can be removed from the crystalling fat is determined by the design of the crystalliser, notably, the type of cooling device either helicoidal or vertical coil or even vertical cooling plates, the cooling exchange surface per unit of volume of fat and the type of agitator.

The nucleation and crystal growth are carefully controlled through the control of temperature

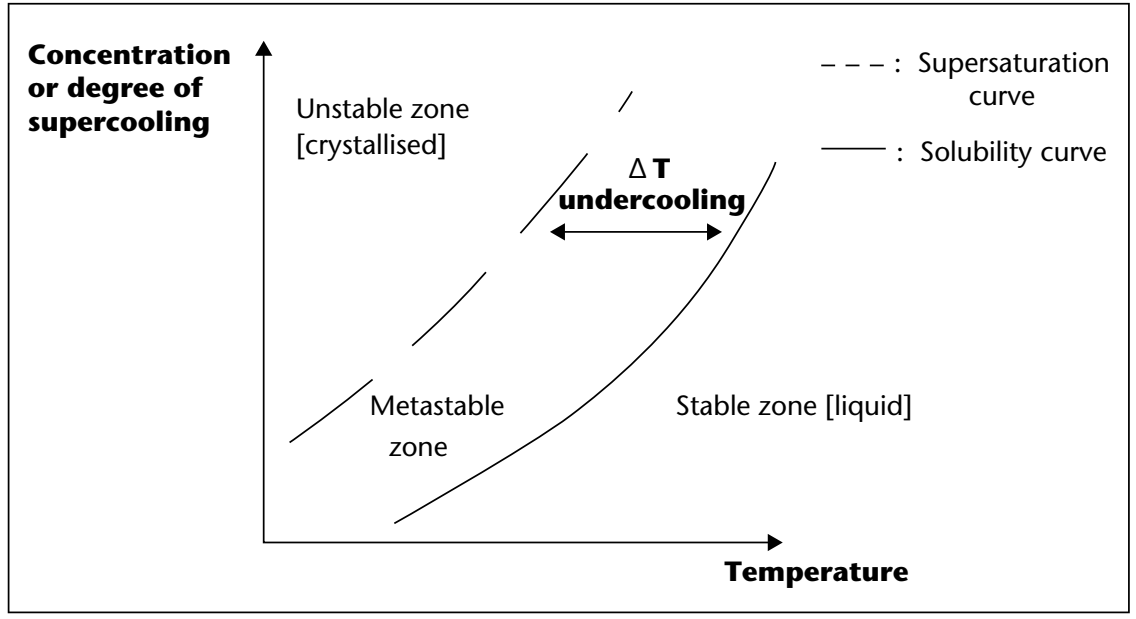

Figure 17. Diagram: degree of supercooling versus temperature

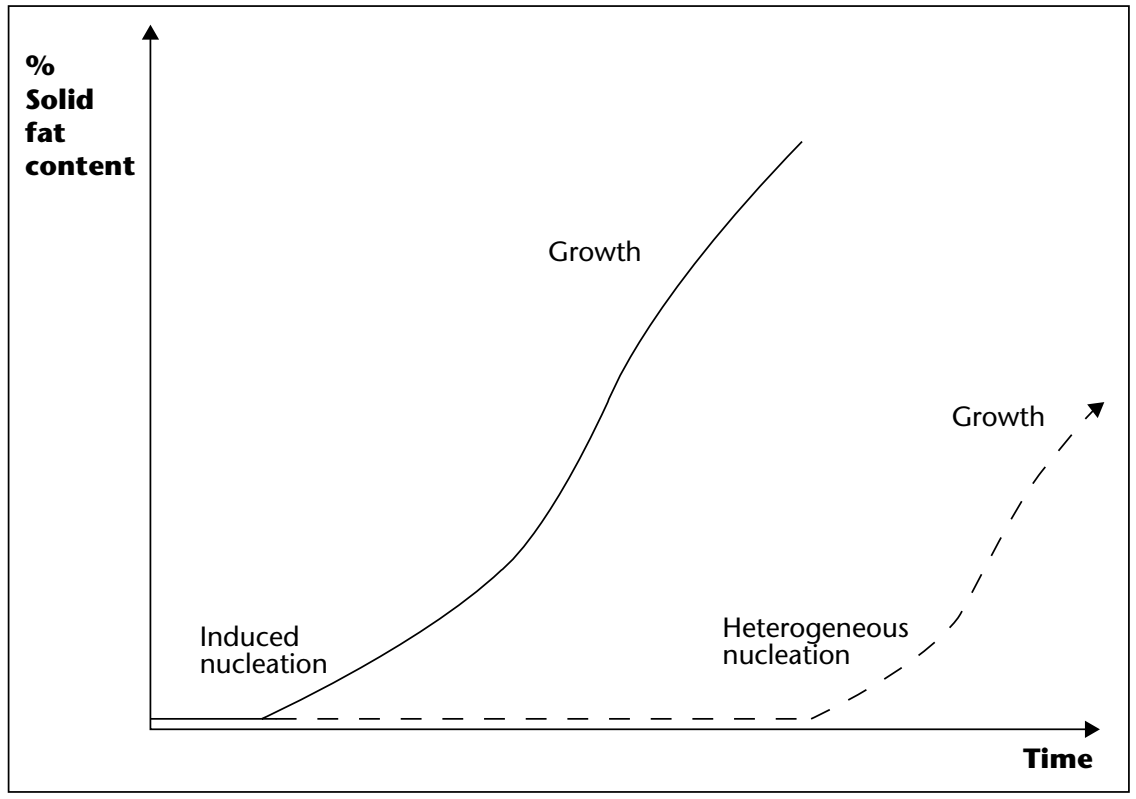

Figure 18. Crystallisation rate of palm olein versus type of nucleation or cooling rate and the agitation rate which are the main processing conditions.

Different separation techniques are available to fractionate the slurry into stearin and olein. Formerly the vacuum drum filter or the horizontal vacuum stainless steel belt filter was employed. Vacuum filtration has long been superseded by the filtration under pressure. Launched in around 1984, the membrane filter press has improved greatly the separation efficiency. Although higher olein yield and harder stearin cake can be achieved while notably playing with the squeezing pressure and the thickness of the chamber, it must be stressed that olein yield still depends on crystallisation conditions too. 
The successful development of dry fractionation resulting from the improvement of the crystallisation technique itself over these last twenty years and from the pressure filtration technology has also superseded the detergent fractionation, victim of its effluent problem and the solvent fractionation victim as the former as well of its operating cost.

Another alternative to membrane filter press for softer stearin production, is the centrifuge separation namely for South America where the soft stearin remains a commodity fat for margarine application. Compared to the vacuum filter, the superdecanter is independent of the type, size and morphology of crystals. For low melting fractions, no remelting of soft stearin is registered. Compared to the membrane filter press, the superdecanter offers many advantages: it is a continuous separation, without hold up giving the possibility to switch easily from one feedstock to another. The stearin is pumpable. The equipment is very compact and fully hygienic with the CIP option.

Finally the quality of the fractions namely the olein depends on the separation temperature and its holding time at that temperature. In contrast the quality of the stearin and the yield of both fractions depend on the separation technique, the crystallisation conditions based on cooling rate program and the mode of separation: fractions obtained either in one step, multi-step or from batch mixed.

A last parameter to be mentioned is the seeding. The starting point of crystallisation of any fat is the formation of seeds or nuclei during the cooling of the melted fat. Two energies are involved, the heat of crystallisation from the interface to the solution and the need of energy to overcome the surface tension during crystals formation. Nucleation of lipids is generally natural: nuclei may be generated from high melting TAGs like in palm oil crystallisation but the nucleation of palm olein has most probability to be initiated by impurities acting as actives sites for other TAGs to be incorporated.

Another source of nucleation may be obtained by blending liquid fat with some higher melting fraction used as a crystallisation promoter. Furthermore nucleation can be initiated by promoters of crystallisation like chemicals added as additives or suitable seeds as fat crystals.

Let's focus on recent developments. Thanks to the multi-step fractionation of palm oil (figure 16), it is possible to obtain a wide range of fractions, namely Super olein and Top olein. Both products are in big demand, because their Saturated Fatty Acid (SFA) content is respectively lower than 40 and $30 \%$. They have a good oxidative stability to be used as frying oil and do not contain TFA. However the crystallisation of these two fractions is posing a problem:

- due to its $\mathrm{Cl}$. Pt of $9{ }^{\circ} \mathrm{C}$, the olein does not recrystallise easily for lack of high melting TAGs or simply for lack of seeds;

- due to the presence of DAGs which induce longer nucleation period, slowing the crystallisation rate.
Referring to the typical diagram saturationsupersaturation: concentration versus temperature (figure 17), we observe a stable zone where TAGs remain liquid because melt is not saturated and the supersaturation curve which separates the crystallisation zone from the metastable zone. When cooling rapidly without seeding, crystallisation will take place at the supersaturation curve with a large delta $T$

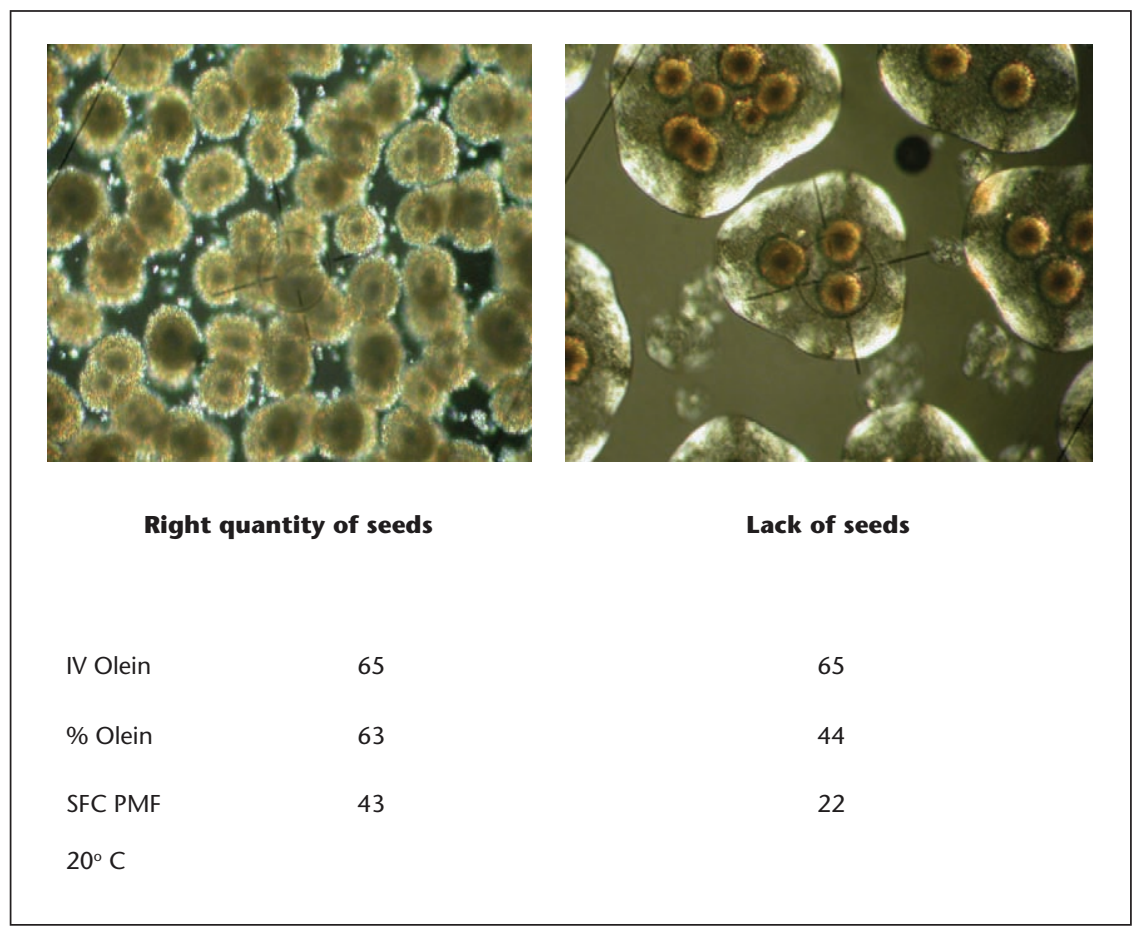

Figure 19. Relationship between quantity of seeds and PMF quality

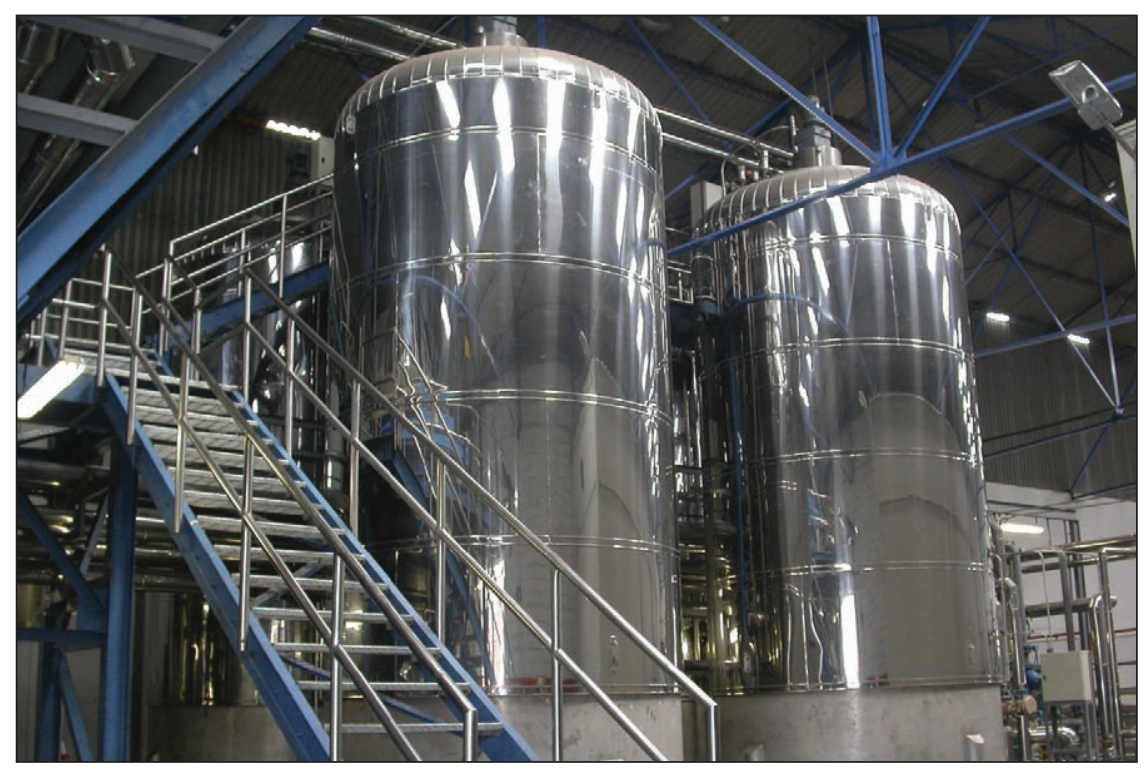

Figure 20. Palm super olein fractionation plant with induced nucleation 
undercooling leading to an excessive nucleation with large number of particules.

In standard conditions, excessive supercooling to speed up the crystallisation might lead to an amorphous crystallisation like a gel for lack of seeds. Consequently in industrial scale, a slight supercooling is applied meaning a long induction time is required because only a heterogeneous nucleation can take place induced by dust or impurities acting as nuclei. Then, the crystal nuclei start to grow further by incorporating TAGs taken from the surrounding liquid phase or solute (figure 18).

Inside the metastable zone, crystallisation is possible but will not occur spontaneously without assistance. Hence the crystallisation of liquid can be initiated alternatively by a special seeding induction taking place in the metastable zone. As a result there is less under- cooling, the delta T undercooling is smaller and consequently there is less nuclei leading to bigger size crystals.

Compared to the heterogeneous nucleation, the induced nucleation (figure 18) has shortened greatly the total crystallisation cycle time.

If all parameters are under control with the right quantity of nuclei needed then seeds will grow steadily to generate uniform dense and hard crystals as shown in figure 19 presenting the polarised light picture of crystals. The filterability is excellent and squeezing pressure may go up to 30 Bar in industrial scale.

For comparison, for the same feed olein crystallised differently, we observe that seeds or nuclei have growth to a certain stage to form spherulites but suddenly the spherulites agglomerate and are surrounded by olein crystallising in amorphous form, called also vitreous phase.
The reason why we observe such break in the crystals growth is due to lack of seeds (figure 19). In both cases, we may obtain 65 IV superolein but the results are totally different in term of olein yield and PMF quality.

The seeding by induced nucleation is very beneficial for superolein and top olein production:

The technology controls the nuclei quantity hence the crystals size. As a result, there is a better selectivity and better olein yield. Due to the quality of crystals, the filtration is faster too. It reduces drastically the crystallisation time hence the capacity of the batch fractionation plant is more than doubled (figure 20).

Last and not least, the induced nucleation enables a saving in energy and investment. 